\title{
Study of the demixing transition in model athermal mixtures of colloids and flexible self-excluding polymers using the thermodynamic perturbation theory of Wertheim
}

\author{
Patrice Paricaud, Szabolcs Varga, ${ }^{\text {a) }}$ and George Jackson ${ }^{\text {b) }}$ \\ Department of Chemical Engineering and Chemical Technology, South Kensington Campus, Imperial \\ College London, London SW7 2AZ, United Kingdom
}

(Received 7 January 2003; accepted 10 February 2003)

\begin{abstract}
Fluid phase separation in model athermal mixtures of colloids and polymers is examined by means of the first-order thermodynamic perturbation theory of Wertheim [M. S. Wertheim, J. Chem. Phys. 87, 7323 (1987); W. G. Chapman, G. Jackson, and K. E. Gubbins, Mol. Phys. 65, 1057 (1988)]. The colloidal particles are modeled simply as hard spheres, while the polymers are represented as chains formed from tangent hard-sphere segments. In this study the like (colloid-colloid, polymerpolymer) and unlike (polymer-colloid) repulsive interactions are treated at the same level of microscopic detail; we do not employ the common Asakura-Oosawa (AO) approximations which essentially involve treating the polymer as an ideal (noninteracting) chain. The effect of varying both the chain length and the diameter of the hard-sphere segments of the polymer on the fluid phase behavior of the model polymer-colloid system is investigated. We focus our attention on the stability of the fluid phase relative to a demixing transition into colloid-rich and polymer-rich fluid phases by using a spinodal instability analysis and determine the full coexistence boundaries (binodal). The colloid-polymer system represents the limit where the diameter of the colloid is much larger than the diameter of the segments making up the polymer chain. The precise segment/ colloid diameter ratio at which liquid-liquid demixing first occurs is examined in detail as a function of the chain length of the polymer. In the case of moderately short chains the addition of polymer induces the "colloidal vapor-liquid" transition found in polymer-colloid systems, while for long chains a "polymeric vapor-liquid" transition is found. The diameter of the polymeric segments must lie between the AO limit (minimum diameter) and the so-called protein limit (maximum diameter) in order for the system to exhibit fluid-fluid phase separation. The maximum value of the segment diameter which induces phase separation is determined from a simple approximate stability analysis. The critical density of the demixing transitions is not found to tend to be zero for infinitely long polymers, but has a limiting value which depends on the diameter of the segment. An examination of the thermodynamic properties of mixing indicates that the fluidfluid phase separation in such systems is driven by a large positive enthalpy of mixing which is induced by a large positive volume of mixing due to the unfavorable polymer-colloid excluded volume interactions. The enthalpy of mixing makes an unfavorable contribution to the overall Gibbs free energy (which is seen to counter the favorable entropy of mixing), giving rise to fluid-fluid immiscibility. () 2003 American Institute of Physics. [DOI: 10.1063/1.1565104]
\end{abstract}

\section{INTRODUCTION}

The theme of this contribution is an examination of the nature of fluid phase separation in purely repulsive models of colloid-polymer systems. In the case of mixtures of simple molecules which interact with dispersive attractive interactions, an unfavorable enthalpy of mixing due to relatively weak unlike interactions is responsible for liquid-liquid phase separation. The long-established thermodynamic explanation for the demixing is that the unfavorable (positive) enthalpic contribution to the free energy increasingly dominates the favorable entropy of mixing as the temperature is

\footnotetext{
a) Present address: Department of Physics, University of Veszprém, H-8201 Veszprém, P.O. Box 158, Hungary.

b) Author to whom correspondence should be addressed. Electronic mail: g.jackson@imperial.ac.uk
}

lowered; the free energy thus increases with decreasing temperature, leading to an instability of the fluid relative to a demixed state. ${ }^{1}$ On the other hand, there is a compelling body of more recent experimental and theoretical work which provides unequivocal evidence for "entropy-driven" fluid phase separation in purely repulsive, athermal, systems (e.g., see the reviews by Frenkel ${ }^{2}$ ). It is now widely accepted that fluid phase separation can occur in binary mixtures of hard repulsive particles if the shapes and/or the sizes of the components are very different. In these athermal systems the internal energy of the system is zero so that the only contribution to the Helmholtz free energy is entropic; it is important to recognize that in the case of phase transitions at constant pressure an enthalpic contribution to the Gibbs free energy may also be important. Contrary to some preliminary theoretical predictions ${ }^{3,4}$ a simple binary mixture of large and 
small hard spheres does not undergo a phase transition between two fluid phases, irrespective of the diameter ratio, as it is found to be metastable with respect to a first-order fluid-solid transition. ${ }^{5,6}$ Fluid phase separation is, however, possible in multicomponent (polydisperse) hard-sphere mixtures for appropriate choices of the size distributions, ${ }^{7,8}$ with the additional possibility of coexistence between two solid phases. ${ }^{9}$

The case of colloid-polymer mixtures, where to a reasonable level of approximation the colloidal particles can be thought of as large hard spheres and the polymers as chains of small hard-sphere segments, is more complex and perhaps more interesting. Such an athermal system can exhibit phase separation into colloid-rich and polymer-rich fluid phases (the review by Poon and Pusey ${ }^{10}$ provides an excellent introduction to the topic). This type of phase behavior has now been studied experimentally in a variety of systems ranging from comparatively simple latex-polystyrene and silicapolydimethylsiloxane colloidal dispersions to biological systems containing proteins or DNA (e.g., see Refs. 10-20). The tendency of the system to reduce the excluded volume interaction between the polymer coils and the colloidal spheres, which then leads to a larger free-volume (translational) entropy, induces an effective attractive (depletion) interaction between the colloidal spheres; this attractive interaction in turn leads to a fluid phase separation into low- and high-concentration colloidal phases which closely resembles the vapor-liquid transition in simple molecules with dispersive interactions. The nature of the effective interaction was first described by Asakura and Oosawa ${ }^{21}$ and then by Vrij ${ }^{22}$ and Joanny et al. ${ }^{23}$ In the case of polymers with overall dimensions which are small compared to the colloid, the colloid-colloid attractive interaction is pairwise additive ${ }^{24,25}$ (and as a consequence is easy to treat theoretically); for polymer dimensions which are of the order of the size of the colloid or larger, pairwise additivity can no longer be assumed. ${ }^{24,26}$ Depending on the relative size and the concentration of the polymer, the effective attractive interaction between the colloids can be tuned, resulting in a rich variety of phases, including both fluid and solid states.

It was recognized early on that the polymer-polymer interaction can be neglected at a first level of approximation (ideal chain) and a qualitative description of the phase behavior of polymer-colloid mixtures can be achieved solely with the incorporation of the colloid-colloid and colloidpolymer hard-body excluded volume interactions. ${ }^{21,22}$ Moreover, if the colloid-polymer interaction is modeled by treating the polymer coil as an effective hard sphere, which corresponds to the so-called Asakura-Oosawa (AO) model, a knowledge of the polymer-colloid size ratio $q=R_{g} / R_{C}$ (where $R_{g}$ is the polymer radius of gyration and $R_{C}$ is the radius of the colloid) is sufficient to describe the main features of the phase behavior. The first theoretical studies of the global phase diagram of the AO model were undertaken by Gast et al. ${ }^{27}$ and by Vincent and co-workers ${ }^{28}$ for mixtures of colloidal hard spheres and noninteracting polymers in a background solvent using a standard perturbation theory for the depletion potential (see Ref. 29 for a review of these types of theoretical approaches). The partitioning of the polymeric component was not considered in these preliminary studies, but was later incorporated into the description of the AO colloid-polymer model by Lekkerkerker et al. ${ }^{30}$ who employed a free-volume theory; this provides the correct description of the fluid-fluid-solid three-phase coexistence region. In the case of short polymers and large colloidal particles corresponding to $q<1$ (ideal polymer limit), the free-volume treatment of the AO model provides a good representation of the simulation data ${ }^{24,25,31}$ and has been validated by an exact one-dimensional description. ${ }^{32-34}$

As the size of the polymer increases relative to the colloid $(q>1)$, the validity of the approximations inherent in the AO becomes questionable; this does not of course imply that the segments making up the chain are larger than the colloid, only that the overall polymer dimension is large. For long polymer chains the interactions between polymer chains are no longer negligible and the overall shape of the polymer is not globular as far as its interaction with colloid is concerned. Sear ${ }^{35}$ has addressed the latter problem by extending the AO treatment to noninteracting chains with dimensions that are larger than the colloidal hard spheres $(q>1)$. In his approach the ideal polymer is described as a number of spherical blobs, $n_{b}$, obtained by scaling the polymer dimension with the size of the colloidal particle $\left(n_{b} \sim R_{g}^{2} / R_{C}^{2}\right)$; the polymer-colloid interaction can then be treated as an excluded volume interaction between a hard-sphere colloid and a chain of $n_{b}$ hard-core spherical blobs of the same diameter as the colloid. Sear treats the contribution of the colloidpolymer interaction to the free energy by using the first-order perturbation theory (TPT1) of Wertheim; ${ }^{36,37}$ we also use the Wertheim TPT1 description, but in our study the polymerpolymer interactions are included explicitly. Sear is thus able to describe the nonspherical nature of the colloid-polymer interaction in the case of long chains for which the standard AO model is inadequate. As with the usual AO model, the system exhibits a fluid-fluid demixing transition between colloid-rich and polymer-rich phases; ${ }^{35}$ the main finding of the work is that the critical density of the colloid decreases and tends to zero as the length of the polymer is increased. We shall return to this point later in the discussion. Schmidt and Fuchs ${ }^{38}$ have also examined the case of polymer dimensions which are larger than the colloid by replacing the hardspherical repulsion between the polymer coil and the colloid by a repulsive step function potential to allow for the possibility of chain configurations where the colloids are "inside" the polymer. The main drawback of this model (and that of Sear ${ }^{35}$ ) is that the interaction between polymers is assumed to be ideal.

Up until this point we have only discussed the description of colloid-polymer systems where the polymer is treated as a noninteracting chain. There has also been recent effort in incorporating the polymer-polymer interactions into the treatment of such systems (the reader is directed to the recent review by Fuchs and Schweizer ${ }^{39}$ for more details, where a particular emphasis is placed on the integral equation approaches). Warren et $a l^{40}$ were one of the first to examine the effect of polymer-polymer interactions on the phase behavior of polymer-colloid systems using a perturbation theory around the $\theta$-point conditions. Integral equa- 
tion approaches have been used extensively to treat the thermodynamic and structural properties of colloid-polymer systems. ${ }^{41,39}$ Sear $^{42}$ has recently used his rescaling approach (representing the polymer which is larger than the colloid as a chain of spherical blobs) to take into account polymerpolymer and polymer-colloid repulsive interactions at the level of the second virial coefficient within a FloryHuggins-type theory; the colloid-colloid interactions are neglected in this study. A discussion of the effect of varying the solvent quality on the phase behavior is also made. The incorporation of polymer-polymer interactions, even at the crude second virial level, leads to the prediction that the density of the monomers at the critical point tends to a finite value as the polymer is made longer, a contrary finding to that of studies with noninteracting polymers. ${ }^{35}$ Although the qualitative trends obtained in simulation studies ${ }^{43}$ are described correctly with the Flory-Huggins-like approach, the critical densities are not in close agreement with the simulation data; this is most likely due to the truncation of the free energy at the level of the second virial coefficient, which essentially means that the treatment will only be valid at low densities. Another particularly appealing approach referred to as the "polymer as soft colloids" model is worth a separate mention: ${ }^{44}$ the polymer-polymer interactions are incorporated by using an effective potential acting between the centers of mass of the chains which enables large-scale simulations of the system. This coarse-graining method has been recently employed by Bolhuis et al. ${ }^{45}$ to study the phase behavior of colloid-polymer mixtures. Simulations of selfavoiding walks were undertaken for pure polymers and their mixtures with a single colloidal hard sphere to compute the polymer-polymer and polymer-colloid center-of-mass radial distribution functions; the structural information can then be inverted by using the hypernetted chain integral equation to determine the effective polymer-polymer and polymer-colloid interaction potentials, which are parametrized in terms of a sum of Gaussians or exponentials. The spherically symmetric effective interactions are thus obtained for a series of polymer dimensions $(q)$ and concentrations and used to simulate the full phase behavior of the colloidpolymer system. As expected, the effect of the polymerpolymer interaction is small for the systems of short chains $(q=0.34)$ and there is close agreement with the results of AO model and free-volume theory. ${ }^{30}$ When the polymers dimension is increased the effect of the polymer-polymer interaction becomes more important and significant qualitative differences are seen with the AO description. As has been predicted by Aarts et al. ${ }^{46}$ with a recent extension of the free-volume theory to systems of interacting polymers, the extent of the fluid-fluid immiscibility is found to decrease on incorporating the polymer-polymer interactions. Bolhuis et $a{ }^{45}$ also show that the colloid density at the critical point is found to be insensitive to the length of the polymer in agreement with the Flory-Huggins findings of Sear. ${ }^{42}$ The main disadvantage of the use of these types of spherically symmetric effective interactions is that the approach is inadequate in describing polymer chains which are large compared to the colloid and that it is restricted to moderate densities of polymer.
Before we outline the main goals of our study it is important to acknowledge the work on the effect of the spherical particles on the dimension of a polymer coil. It is well recognized that a polymer adopts a more extended configuration in a "good" solvent and a more collapsed configuration in a "bad" solvent (which can lead to demixing). ${ }^{47-49}$ The collapse of the polymer dimension does not necessarily imply phase separation, but is clearly closely related to it. The evidence of a polymer collapse transition in simulation studies for purely repulsive (athermal) models where the solvent and segments making up the polymer are of the same size is rather inconclusive $;^{50-53}$ one should point out that these are models of polymer solutions and not colloidpolymer systems where the colloid is much larger than the monomeric segments making up the polymer. Taylor and Lipson $^{54}$ have used the Yvon-Born-Green integral equation approach for hard-sphere and hard-sphere models of polymer solutions in which the spherical segments are all the same size to show that the chain contraction with increasing solvent density is almost identical to what one would expect in the pure polymer melt. The lack of a dramatic collapse of the polymer is consistent with a polymer in a good solvent, which would support the conclusion that the system does not undergo a fluid phase transition (Taylor and Lipson ${ }^{54}$ did not examine the fluid phase equilibria in their study). The lack of a demixing transition in such an athermal system has been recently corroborated by studies of the fluid phase equilibria in model polymer solutions with the Wertheim TPT1 description: ${ }^{55}$ the athermal mixture is stable and does not demix regardless of the length of the hard-sphere chain. In the case of polymer solutions the fluid phase separation is driven by the polymer-solvent attractive interactions. ${ }^{55}$ As was mentioned earlier, there is clear evidence of the existence of polymer collapse and a demixing transition for athermal systems in which the diameter of the spherical particle (in our case the colloid) is considerably larger than that of the segments making up the chain. For these athermal models of colloid-polymer systems a collapse of the polymer dimension is unambiguously observed when the concentration of the colloid hard spheres is increased. ${ }^{24,56}$ The situation is completely different when attractive interactions are present: chain collapse is found for a symmetric model in which the sphere-sphere, sphere-segment, and segmentsegment size and interaction energies are all equivalent, ${ }^{57-61}$ implying liquid-liquid immiscibility in such a system. Less work has been undertaken on the incorporation of attractive interactions in models of colloid-polymer systems in which the sphere-segment size ratio is large (e.g., see Ref. 62). We do not discuss the effect of attractive interactions on the phase behavior further, as the focus of our paper is on the fluid-phase separation of athermal hard-core models of both the colloid and polymer.

In our contribution we study the fluid phase equilibria of mixtures of large hard spheres (colloid) and chains formed from smaller hard-sphere segments (polymer). The density range over which one would find fluid-solid phase transitions in such systems is not considered. In contrast to the other theoretical work mentioned earlier we study a "microscopic" model of the polymer in which both the polymer- 
polymer and polymer-colloid excluded volume interactions are treated at the level of the monomeric segments making up the chain. The polymer dimension (radius of gyration) does not enter into the description in an explicit manner. In principle, this means that our approach can deal with the situation in which the polymer is smaller or larger than the colloidal particle. The Wertheim TPT1 approach $^{36,37}$ is used to describe the polymer-polymer and polymer-colloid contributions to the free energy; this choice is made as the theory provides an excellent description of the equation of state of chains of tangent hard spheres and mixtures. ${ }^{37,63} \mathrm{By}$ using a spinodal stability analysis similar to that employed in an examination of isotropic-isotropic demixing in mixtures of cylindrical particles of different length and shape (diameter), ${ }^{64}$ we examine the global fluid phase diagram of the model colloid-polymer system. The precise chainsegment to colloid diameter ratio that gives rise to an instability in the fluid (and leads to fluid-fluid demixing) is determined for varying lengths of the polymer chain. The full fluid-phase behavior (binodals) is also determined for the system. We relate our findings to existing theoretical predictions, but leave any direct comparison with experimental data to a future publication. In addition, an analysis of the thermodynamics of mixing in our athermal colloid-polymer model is made to highlight the roles of the entropic and enthalpic contributions to the free energy in such systems.

\section{WERTHEIM TPT1 APPROACH}

We use the Wertheim thermodynamic perturbation theory (TPT1 $)^{36,37}$ to study the global fluid phase behavior of the binary mixture of colloids and polymer chains as it provides a direct insight into the effect of varying the size of the segment diameter and the chain length. As was mentioned earlier, the theory allows one to treat the system at the level of the monomeric segments. Both the colloidal particles and the segments making up the chain are modeled as a hard spheres of diameter $\sigma_{C}$ (colloid) and $\sigma_{P}$ (polymer segment). The chains are assumed to be formed from $m$ tangentially bonded segments, but are otherwise completely flexible. The interaction between the colloidal hard-sphere and a polymer hard-sphere segment is taken to be additive, i.e., $\sigma_{C P}=\left(\sigma_{C}\right.$ $\left.+\sigma_{P}\right) / 2$. The pair potential between the different species can be written in a compact form as

$$
u_{i j}\left(r_{i j}\right)=\left\{\begin{array}{cc}
\infty, & r_{i j}<\sigma_{i j}, \\
0, & r_{i j} \geqslant \sigma_{i j},
\end{array}\right.
$$

where $i, j=P, C$ and $r_{i j}$ is the distance between the centers of the two spheres (colloid-colloid, colloid-monomer, and monomer-monomer).

Within the TPT1 approach the Helmholtz free energy of this binary colloid-polymer mixture can be written as a sum of ideal $A^{\text {ideal }}$, hard-sphere $A^{\mathrm{HS}}$, and chain formation $A^{\text {chain }}$ terms:

$$
\frac{\beta A}{N}=\frac{\beta A^{\text {ideal }}}{N}+\frac{\beta A^{\mathrm{HS}}}{N}+\frac{\beta A^{\text {chain }}}{N},
$$

where $\beta=1 / k T$ ( $T$ is the temperature and $k$ is Boltzmann's constant) and $N$ is the number of particles (sum of the $N_{C}$ colloids and $N_{P}$ polymers). The ideal free energy of the binary mixture is given by

$$
\frac{\beta A^{\text {ideal }}}{N}=\sum_{i=C, P} x_{i}\left(\ln \rho-1+\ln v_{i}+\ln x_{i}\right),
$$

where $\rho=N / V$ is the number density, $x_{i}$ is the mole fraction of the component $i$, and $v_{i}$ is the de Broglie volume, which takes into account the translational (and rotational in the case of the polymer) contributions to the kinetic energy of each component; though the precise form of the de Broglie volume is not important in studies of phase equilibria, it is included for completeness. The ideal entropy of mixing is seen as the last term of Eq. (3).

An accurate and relatively simple expression for the residual free energy of a fluid mixture of hard spheres of different diameter is given by Boublik ${ }^{65}$ and Mansoori et al. ${ }^{66}$ as

$$
\frac{\beta A^{\mathrm{HS}}}{N}=\frac{6}{\pi \rho}\left[\left(\frac{\zeta_{2}^{3}}{\zeta_{3}^{2}}-\zeta_{0}\right) \ln \left(1-\zeta_{3}\right)+\frac{3 \zeta_{1} \zeta_{2}}{\left(1-\zeta_{3}\right)}+\frac{\zeta_{2}^{3}}{\zeta_{3}\left(1-\zeta_{3}\right)^{2}}\right] .
$$

In the case of our colloid-polymer binary mixture the reduced densities are defined as $\zeta_{1}=(\pi \rho / 6)\left(x_{C} \sigma_{C}^{l}\right.$ $\left.+m x_{P} \sigma_{P}^{l}\right)$, where $l=0-3$; the chain length is included in the definition of the reduced density as the number of monomeric segments making up the chain is given by $N_{\text {seg }}=m N_{P}=m x_{P} N$. The total packing fraction of the system is given by $\eta=\zeta_{3}=(\pi \rho / 6)\left(x_{C} \sigma_{C}^{3}+m x_{P} \sigma_{P}^{3}\right)$. The Boublik-Mansoori description has been shown to provide an accurate representation of the equation of state for binary mixtures of hard spheres of significantly different size (corresponding to diameter ratios of up to 20:1) over a wide range of compositions; ${ }^{67}$ this is a particularly relevant point for our model colloid-polymer systems where the diameter of the polymer segment is much smaller than the colloid. The expression reduces to the accurate Carnahan-Starling ${ }^{68}$ equation for hard spheres in pure fluid limits.

The remaining contribution to the free energy due to the bonding together of the $m$ hard-sphere monomeric segments to form the tangent, flexible polymer chain merits a more detailed discussion. Wertheim ${ }^{69}$ originally developed his first-order perturbation theory to describe the thermodynamic properties of associating fluids. By examining a two-site associating monomeric fluid, Wertheim ${ }^{36}$ subsequently obtained an expression for the free energy of the chain fluid simply in terms of the structure of the monomer hard-sphere reference fluid and the average chain length of the polydisperse mixture of chain aggregates that are formed on association. An identical expression for the free energy of the monodisperse hard-sphere chain fluid can be obtained by considering a mixture of associating hard spheres in the limit of complete association; ${ }^{37}$ in addition, the treatment was extended to provide a general equation of state for mixtures of chain molecules (and the special case of mixtures of spheres 
and chains which are the subject of our contribution). ${ }^{37}$ In the Wertheim first-order theory, bonding at one site is assumed to be independent from bonding at another site. As a consequence, differences in the conformation of the chain or in the degree of flexibility cannot be treated at this level of approximation. The effect of flexibility can be incorporated by extending the theory to second order, ${ }^{36}$ but this is beyond the scope of our work. The Helmholtz free energy due to linking $m$ monomeric hard-sphere segments together at contact in the presence of colloidal hard-sphere particles can be expressed in terms of the contact value of the reference monomer-monomer pair correlation function as ${ }^{37}$

$$
\frac{\beta A^{\text {chain }}}{N}=-x_{P}(m-1) \ln g_{P P}^{\mathrm{HS}}\left(\rho, x ; \sigma_{C}, \sigma_{P}, m\right) .
$$

In order to be consistent with the treatment of the reference hard-sphere contribution to the free energy, the Boublik ${ }^{65}$ expression for the contact value of the pair correlation function of like hard spheres in a mixture of hard spheres of different size is used in expression (5):

$$
\begin{aligned}
g_{P P}^{\mathrm{HS}}\left(\rho, x ; \sigma_{C}, \sigma_{P}, m\right)= & \frac{1}{\left(1-\zeta_{3}\right)}+\frac{3 \sigma_{P}}{2} \frac{\zeta_{2}}{\left(1-\zeta_{3}\right)^{2}} \\
& +\frac{\sigma_{P}^{2}}{2} \frac{\zeta_{2}^{2}}{\left(1-\zeta_{3}\right)^{3}} .
\end{aligned}
$$

An alternative derivation of expression (5) which adds some insight into the approximations that are involved in the TPT1 treatment can be obtained by considering the chemical potential of a chain in a reference solvent of monomers. ${ }^{70-75}$ Some time before Wertheim, Ben-Naim ${ }^{70}$ derived an exact expression for the chemical potential $\mu_{P}$ of a single chain (infinitely dilute) in terms of the $m$-body cavity function $y_{m}^{\text {ref }}(1, \ldots, m)$ the reference monomers as

$$
\beta \mu_{P}=-\ln y_{m}^{\mathrm{ref}}(1, \ldots, m),
$$

where the notation $(1, \ldots, m)$ is used to denote the relative positions of all of the segments in the chain. Equation (7) can be thought of as a definition of the cavity function. In the case of chains of hard-sphere segments tangentially bonded in rolling contact, the chemical potential can be expressed in terms of the $m$-body distribution function $y_{m}^{\mathrm{HS}}(1, \ldots, m)$ $=g_{m}^{\mathrm{HS}}(1, \ldots, m) \exp \left(\beta u_{m}^{\mathrm{HS}}\right)=g_{m}^{\mathrm{HS}}\left(\sigma_{12} \sigma_{23} \cdots \sigma_{m-1, m}\right)$ of the hard-sphere monomers for the contact distances $\sigma_{i j}$ :

$$
\beta \mu_{P}=-\ln g_{m}^{\mathrm{HS}}\left(\sigma_{12} \sigma_{23} \cdots \sigma_{m-1, m}\right) .
$$

Two approximations now have to be made to make use of this exact result for the chemical potential of a infinitely dilute chain to provide a description of the free energy of the system. If one assumes that the chemical potential for the formation of subsequent chains in the mixture is the same as that for the infinitely dilute solution, the contribution to the Helmholtz free energy of forming $N_{P}$ polymer chains can be approximated by

$$
\beta A^{\text {chain }} \approx-N_{P} \ln g_{m}^{\mathrm{HS}}\left(\sigma_{12} \sigma_{23} \cdots \sigma_{m-1, m}\right),
$$

$$
\frac{\beta A^{\text {chain }}}{N} \approx-x_{P} \ln g_{m}^{\mathrm{HS}}\left(\sigma_{12} \sigma_{23} \cdots \sigma_{m-1, m}\right) .
$$

The $m$-body distribution function can then be treated at the level of a linear approximation such that it factorizes into a product of $(m-1)$ equivalent monomer-monomer pair contributions:

$$
\begin{aligned}
g_{m}^{\mathrm{HS}} & \left(\sigma_{12} \sigma_{23} \cdots \sigma_{m-1, m}\right) \\
& \approx g_{2}^{\mathrm{HS}}\left(\sigma_{12}\right) g_{2}^{\mathrm{HS}}\left(\sigma_{23}\right) \cdots g_{2}^{\mathrm{HS}}\left(\sigma_{m-1, m}\right) \\
& =g_{P P}^{\mathrm{HS}}\left(\sigma_{P}\right)^{(m-1)} .
\end{aligned}
$$

On substituting Eq. (10) for the $m$-body distribution function into expression (9) for the free energy, one recovers the TPT1 expression (5):

$$
\frac{\beta A^{\text {chain }}}{N} \approx-x_{P} \ln g_{P P}^{\mathrm{HS}}\left(\sigma_{P}\right)^{(m-1)}=-x_{P}(m-1) \ln g_{P P}^{\mathrm{HS}}\left(\sigma_{P}\right) .
$$

The significance of the linear approximation to the $m$-body distribution function is that one loses information about the structure of the chain, including the lack of an explicit description of the end-to-end vector and the radius of gyration which are used as measures of the dimension of the polymer. This does not mean, however, that the polymer dimension is absent from the Wertheim TPT1 description. When the TPT1 free energy is expanded as a virial series in density, the resulting virial coefficients are found to be dependent on the chain length $m .^{76}$ The ultimate consequence of this for our colloid-polymer model is that both the polymer-polymer and polymer-colloid TPT1 excluded volumes will depend on the chain length, i.e., the dimension of the polymer. The TPT1 second virial coefficient (excluded volume) turns out to be a linear function of $m$ (Refs. 76 and 77); this corresponds to the correct scaling for long linear chains, ${ }^{77}$ but not for fully flexible chains in a good solvent where the second virial coefficient scales as $m^{3 v} \sim m^{1.5}$ (see Refs. 49 and 76). A simple correction can be added to the TPT1 treatment to account for the correct second virial coefficient of the fully flexible chains, though only a slight improvement in the description of the equation of state is expected. ${ }^{76}$

The Wertheim TPT1 theory described here was developed to deal with the fluid state of mixtures of chain molecules, and no description of the solid states was incorporated into the treatment. Previous work with athermal colloid-polymer systems has shown that there are regions corresponding to fluid-solid equilibria which are particularly extensive for colloid-polymer systems comprising short chains at high densities of the colloid (e.g., see Refs. 30, 39, 45, and 46). Although there has been some work on the fluid-solid equilibria of flexible chains of tangent hard spheres within an TPT1-like treatment, ${ }^{78}$ no general TPT1 description is available at this stage for mixtures of large hard spheres and hard-sphere chains. As with some of the other studies, one could assume that the polymer is not playing an important role in determining the structure of the 
solid, but this will only be valid for short polymers with segments that are much smaller than the colloid. In our paper we focus on the effect of the polymer chain length $(m)$ and the segment-colloid size asymmetry (characterized by the diameter ratio $d=\sigma_{P} / \sigma_{C}$ ) on the fluid phase behavior, avoiding the high-density states where one would expect fluid-solid transitions (highlighting states where a stable solid phase would preempt the fluid-fluid coexistence). On examining the TPT1 expressions (3)-(6) that make up the free energy of the athermal colloid-polymer system, it is clear that the thermodynamic properties of the mixture at a given composition (the mole fraction of the polymer $x$ $=x_{P}$ ) and density (reduced number density $\rho^{*}=\rho \sigma_{C}^{3}$ ) only depend on the number of segments in the chain $m$ and the polymer-colloid diameter ratio $d$. One can thus examine the global fluid phase behavior entirely in terms of these two parameters. Having described the analytical form of the Helmholtz free energy for our athermal colloid-polymer model, the TPT1 expressions can easily be used to determine the thermodynamics and the fluid phase equilibria of the system. The other thermodynamic properties are obtained through the standard relations: ${ }^{1}$ pressure $P=-(\partial A / \partial V)_{N, T}$; chemical potential of each component $i, \quad \mu_{i}=\left(\partial A / \partial N_{i}\right)_{N_{j \neq i} V, T} ;$ internal energy $U=$ $-[\partial(A / T) / \partial(1 / T)]_{N, V}$; enthalpy $H=U+P V$; entropy $S$ $=(U-A) / T$; and Gibbs free energy $G=A+P V$ (the last three expressions corresponding to the appropriate Legendre transformations).

\section{RESULTS AND DISCUSSION}

The simplest method to search for the molecular parameters $(d, m)$ which give rise to an instability of the mixed (homogeneous) fluid state relative to a demixed state in the binary colloid-polymer hard-core mixture is based on a determination of the spinodal. The spinodal or limit of stability of the miscible state is obtained from the following condition for the second derivative of the Gibbs free energy $G$ with respect to composition: ${ }^{1}$

$$
\left(\frac{\partial^{2} G / N}{\partial x^{2}}\right)_{P}=0 .
$$

The Gibbs function $G$ is the natural thermodynamic function for phase equilibria in mixtures at constant pressure. The solution of Eq. (12) determines the spinodal curve of the phase diagram, which meets the binodal (phase coexistence) curve at the critical point. In order to locate the critical points of the phase separation, the third derivative of the Gibbs free energy with respect to composition must also vanish: ${ }^{1}$

$$
\left(\frac{\partial^{3} G / N}{\partial x^{3}}\right)_{P}=0 .
$$

For given values of the molecular parameters $(d, m)$ of the colloid-polymer system, Eqs. (12) and (13) together determine the critical density and composition of the fluid-fluid demixing transition if it exists. The two conditions are solved

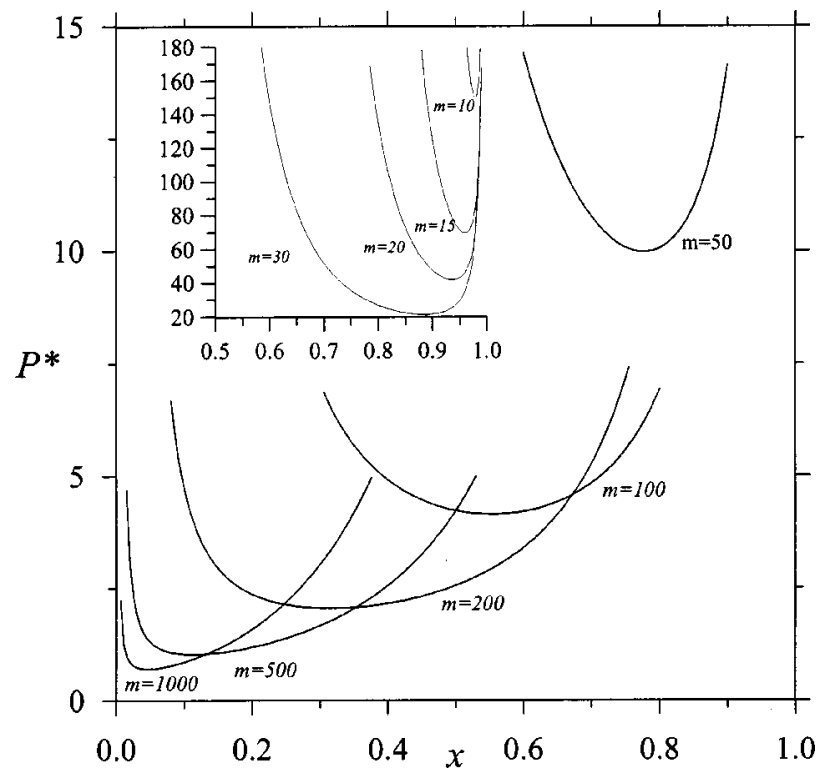

FIG. 1. Pressure-composition representation of the spinodal curves of the fluid-fluid phase equilibria for athermal binary mixtures of colloids (hard spheres of diameter $\sigma_{C}$ ) and flexible polymers (chains formed from tangent hard spheres of diameter $\sigma_{P}$ ) determined from the Wertheim TPT1 approach. Results are presented for systems with a fixed polymer-segment to colloid diameter ratio of $d=\sigma_{P} / \sigma_{C}=0.06$ for polymer chain lengths ranging from $m=10$ up to 1000 . The reduced pressure is defined as $P^{*}$ $=\beta P \sigma_{C}^{3}$, and $x=x_{P}$ represents the mole fraction of the polymers.

numerically by expressing them analytically in terms of the appropriate volume and composition derivatives of the Helmholtz free energy. ${ }^{1}$

From previous studies on athermal mixtures of colloids and polymers and of other hard-body cylindrical molecules ${ }^{64}$ it is well recognized that the size and shape of the two components have to be very different for fluid-fluid demixing to be observed. In such systems the mixing of the two components is associated with an unfavorable unlike packing (excluded volume) entropy. As an initial step, we search for the first sign of an instability in the fluid phase [cf., Eq. (12)] of the colloid-polymer mixture for systems with an increasing polymer chain length $(m=10-1000$ segments), but with a fixed segment/colloid diameter ratio $(d$ $=0.06$ ). If one employs the usual polymer scaling ideas, ${ }^{49}$ the radius of gyration of a polymer in a good solvent scales as $R_{g} \sim m^{1 / 2} \sigma_{P}$, which would correspond to a polymer/ colloid dimension ratio of $q=R_{g} / R_{C} \sim m^{1 / 2} d$ in terms of our parameters. In the case of the systems with chains of length $m=500$ and 1000 , the overall dimension of the polymer is larger than the colloid ( $q \sim 1.3$ and $q \sim 1.9$, respectively), while for systems with $m \leqslant 200$ the polymer dimension is smaller than that of the colloid with $q<1 \quad(q \sim 0.85$ for chains of length $m=200$ and $q \sim 0.20$ for $m=10$ ). With this choice of diameter ratio $(d=0.06)$ and set of chain lengths $m$ one is thus able to cover systems of both short and long polymers relative to the colloid.

The spinodal boundaries denoting the limit of stability of the colloid-polymer mixture with $d=0.06$ are shown in Fig. 1 for polymer chains of varying length. The extent of the fluid-fluid demixing is seen to increase with increasing polymer chain length. The critical pressure $\left(P_{\mathrm{cr}}^{*}=\beta P \sigma_{C}^{3}\right)$ and the 
critical packing fraction $\left(\eta_{\mathrm{cr}}=\pi \rho^{*}\left(1-x+x m d^{3}\right) / 6\right)$ corresponding to the minima of the spinodal curves both decrease with increasing chain length. As the chain length is increased, the composition at critical point also moves from the polymer-rich region of the phase diagram to the colloidrich region. This means that for systems with short chains a "colloidal vapor-liquid" transition is seen between a colloid-poor and a colloid-rich phase (see inset of Fig. 1), while a "polymeric vapor-liquid" transition between a polymer-poor and a polymer-rich phase can be observed for long chains. In other words, the short polymers act as the "depletion agent" and mediate the phase separation between the colloids in one limit, while the colloids play the role of the "depletion agent" in the phase separation of long polymers in the other. This reversal in the role of the colloid and polymer has not been highlighted previously. For polymer chains of moderate length (e.g., $100<m<200$ ) the phase behavior is more symmetric in comparison and neither component can strictly be referred to as the "depletion agent;" one should not use the analogy with a "vapor-liquid" transition for the fluid phase behavior in this case. An additional consequence of this is that neither the polymer nor the colloid should really be taken as the perturbative component to describe the phase separation of the other component in a semigrand ensemble treatment. It is interesting to note that the critical pressure and packing fraction (cf. Fig. 2) do not tend to zero in the limit of a very long polymer chain $(m \rightarrow \infty)$, but converge to finite values: in the case of the colloid-polymer system with $d=0.06$ the infinite-chain limiting values of the critical point are $\eta_{\mathrm{cr}}=0.1$ and $P_{\mathrm{cr}}^{*}=0.31$. This limiting behavior has also been found in the studies of $\mathrm{Sear}^{42}$ and Bolhuis et al. ${ }^{45}$ which explicitly include the polymer-polymer interactions; when the polymer is treated as an ideal chain, the critical density is found to vanish for infinite chain lengths of the polymer. ${ }^{35}$

We now examine the effect of varying the segment to colloid diameter ratio on the stabilization of the fluid-fluid demixing transition. By simultaneously solving the two conditions for criticality [Eqs. (12) and (13)], the critical properties (packing fraction, mole fraction, and pressure) are determined for the colloid-polymer mixture with a given chain length $m$ and diameter ratio $d$. The chain-length dependence of the critical packing fraction for selected diameter ratios is shown in Fig. 2. In all cases a fluid demixing transition is not likely to be found for systems of short chains $(m<15)$, because this would correspond to states with a very high packing fraction ( $\eta_{\mathrm{cr}}>0.5$ at the critical point) where one would expect the solid phases to be stable. On the other hand, the critical packing fraction $\eta_{\text {cr }}$ is seen to decrease rapidly with increasing chain length, and as observed previously, it tends to a limiting value at large $m$. The long-chain limit of $\eta_{\text {cr }}$ is seen to decrease when the diameter ratio is decreased. For the system of polymers with the smallest segments $(d$ $=0.001$ ), the critical packing fraction decays more slowly with chain length and is always found to be higher than that of the system with $d=0.005$ over the range of $m$ shown in the figure. This finding suggests that a decrease in the polymer segment size promotes the demixing transition up to a certain value of $d$, but that any further decrease in segment

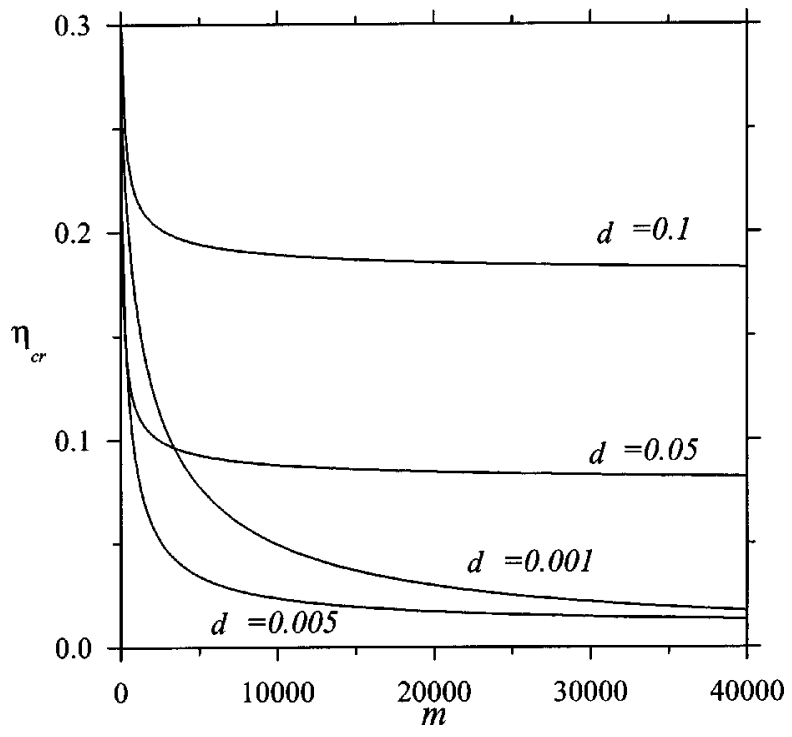

FIG. 2. Critical packing fraction of the fluid-fluid phase equilibria for athermal binary mixtures of colloids (hard spheres of diameter $\sigma_{C}$ ) and flexible polymers (chains formed from tangent hard spheres of diameter $\sigma_{P}$ ) determined from the Wertheim TPT1 approach. Results are presented for selected values of polymer-segment to colloid diameter ratio $d=\sigma_{P} / \sigma_{C}$ as a function of the polymer chain length $m$. The packing fraction is defined as $\eta$ $=\pi \rho^{*}\left(1-x+x m d^{3}\right) / 6$.

size results in a stabilization of the mixed state with a corresponding increase in the critical density. This result is not surprising because as $d$ is made very small (for a fixed chain length $\mathrm{m}$ ) the polymer can be thought of as an ideal gas; there is no fluid phase demixing transition in the limiting ideal-gas +hard-sphere mixture, though there is the usual fluid-solid phase transition at high packing fraction. ${ }^{79}$

The dependence of the critical packing fraction $\eta_{\mathrm{cr}}$ and composition $x_{\mathrm{cr}}$ on the diameter ratio is plotted in Fig. 3(a) for polymers of short, moderate, and long length $(m=10$, 100, and 1000). The observation that the segment size cannot be too low or too high in order for the system to exhibit a stable region of fluid phase separation, either in the polymerrich (small $d$ ) or in the colloid-rich (large $d$ ) regions, is clearer to see in this representation. For values of the critical packing fraction above $50 \%$ one would expect the appearance of solid phases. In the case of the larger diameter ratios $(d>0.2)$, the critical point of the mixture moves deep into the solid region; it is likely that a first-order fluid-solid transition between two colloid-rich phases preempts the "polymeric vapor-liquid" transition, because the pure hard-sphere colloid freezes at around $\eta=0.5$. For systems with small values of $d(d<0.01)$, the critical packing fraction does not increase in the same way as for systems with large $d$. In this case it is probable that there is a "colloidal vapor-solid" transition because the critical pressure is seen to diverge for very small values of $d$ [see Fig. 3(b)]. This is also consistent with the phase diagram of the AO model for small polymers where the size of the polymer (characterized by the radius of gyration) must exceed a certain value $(q>0.3)$ to stabilize the "colloidal vapor-liquid" transition with respect to the "colloidal vapor-solid" transition. ${ }^{30,45}$ In the limit of short chains, $m \rightarrow 1$, the disappearance of the demixing depends 


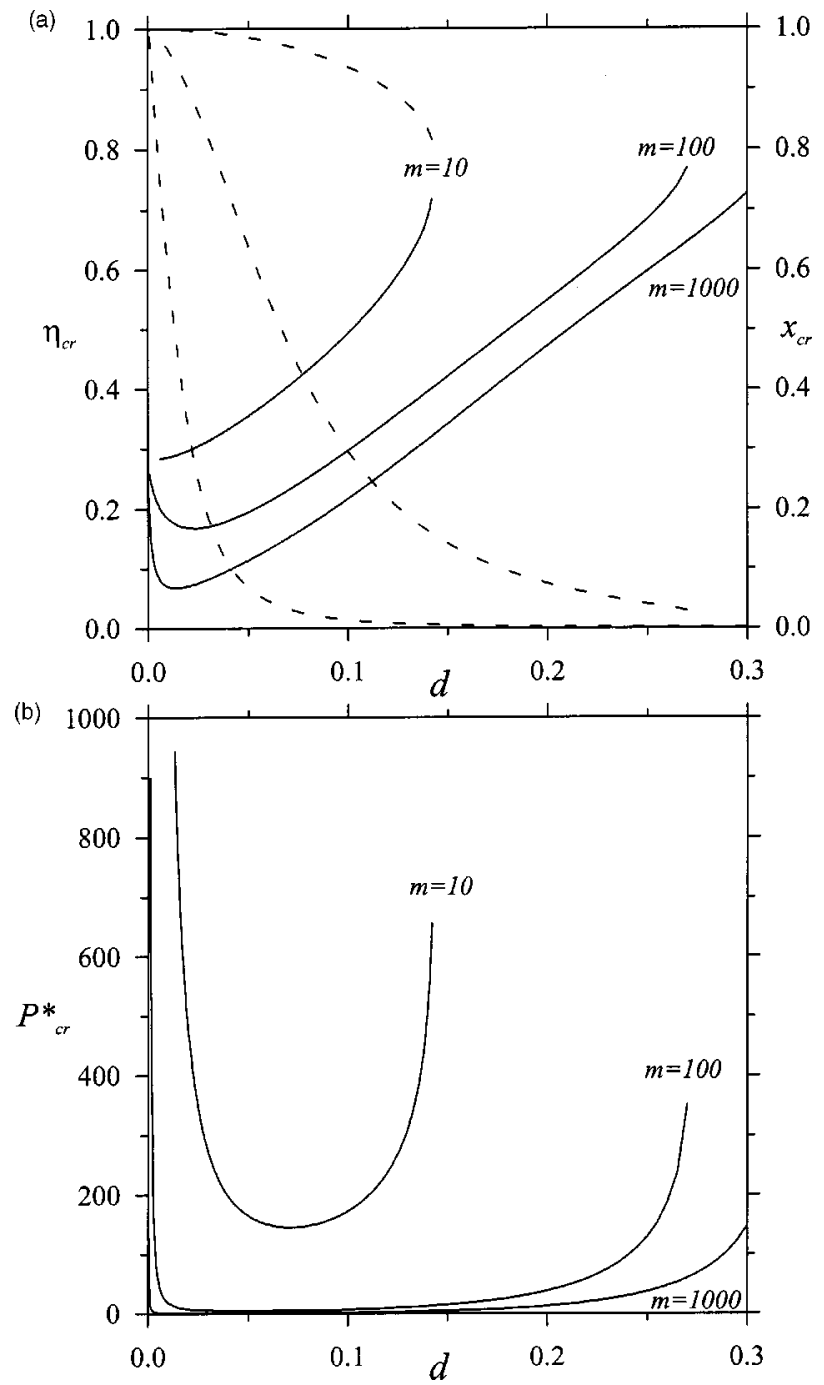

FIG. 3. Dependence of the critical properties on the polymer-segment to colloid diameter ratio $d=\sigma_{P} / \sigma_{C}$ for athermal binary mixtures of colloids (hard spheres of diameter $\sigma_{C}$ ) and flexible polymers (chains formed from tangent hard spheres of diameter $\sigma_{P}$ ) determined from the Wertheim TPT1 approach. The critical packing fraction (continuous curves) and composition (dashed curves) are shown in (a) and the critical pressure in (b) for selected values of the polymer chain length $m$. The reduced packing fraction and pressure are defined as $\eta=\pi \rho^{*}\left(1-x+x m d^{3}\right) / 6$ and $P^{*}=\beta P \sigma_{C}^{3}$, and $x$ $=x_{P}$ represents the mole fraction of the polymers.

strongly on the value of the diameter ratio; on decreasing the diameter ratio, the lowest possible value of $m$ first decreases and later increases again (e.g., when $d=0.1$ the limiting chain length is $m=6.3$, for $d=0.005$ it drops to $m=2.9$, and for $d=0.001$ it increases again to $m=17.0$ ). The trimer thus appears to be the shortest chain which may show a metastable fluid phase and demixing.

In view of the above findings it would be desirable to determine the precise molecular parameters $(d, m)$ for which we would expect our polymer-colloid system to exhibit fluid-solid separation. This is highly nontrivial task because the solid phase is not incorporated into the theory. In this work we make no attempt to determine the Asakura-Oosawa limit (minimum size of the segments making up the polymer characterized by $d$ ), but the protein limit (maximum size of the polymeric segments) can be predicted in the light of

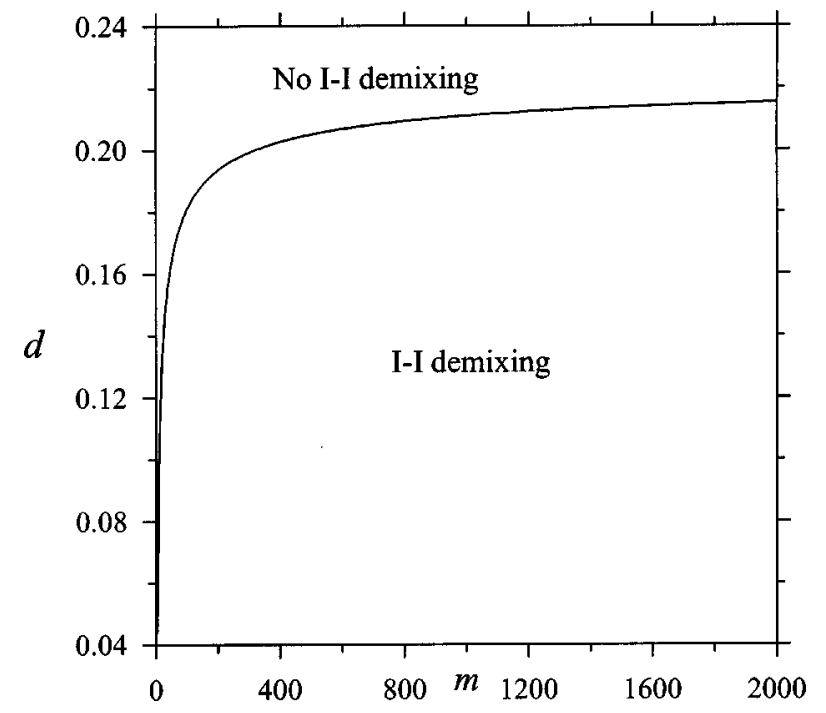

FIG. 4. Maximum value of diameter ratio $d=\sigma_{P} / \sigma_{C}$ for the demixing transition of athermal binary mixtures of colloids (hard spheres of diameter $\sigma_{C}$ ) and flexible polymers (chains formed from tangent hard spheres of diameter $\sigma_{P}$ ) determined from the Wertheim TPT1 approach as a function of the chain length $m$. The term I-I demixing is used to denote a phase separation between two isotropic fluid states.

knowledge about the colloidal solidification. A pure hardsphere system (colloid) undergoes a first-order fluid-solid transition $^{2}$ at $\eta_{F}=0.494$ and $\eta_{S}=0.545$, and a "polymeric vapor-liquid" transition (which occurs in the high-colloidmole-fraction region of the phase diagram) is not likely for the molecular parameters resulting in a critical packing fraction higher than about $50 \%$. To determine the maximum size of the monomers for which the system still exhibits a stable fluid-fluid demixing transition, we assume that the critical packing fraction cannot be higher than $50 \%$. By adding this new constraint $\left(\eta_{\mathrm{cr}}^{\max }=0.5\right)$ to the conditions (12) and (13), the upper limit of the diameter ratio $d$ can be determined for a system with a given chain length $m$. In Fig. 4 we show that the maximum value of the segment diameter increases as the polymer chain is made longer. Furthermore, it is clear that the diameter of the segments making up the chain is always smaller than the diameter of the colloid $(d<1)$. This supports the conclusion ${ }^{54,55}$ that model athermal polymer solutions of hard spheres and chains of hard sphere segments of the same size $(d=1)$ do not exhibit a fluid phase instability with the corresponding demixing. In the case of polymer solutions the attractive interactions play a key role in promoting the fluid phase immiscibility (cloud curves). ${ }^{55}$ The limiting diameter ratio below which one would expect the athermal colloid-polymer model to exhibit fluid phase separation appears to go to a limiting value for infinitely long chains $\left(d_{\infty} \approx 0.22\right)$. This result is consistent with the simulation study of Suen et al. ${ }^{56}$ for a single athermal hard-sphere polymer in a hard-sphere solvent, where the system with $d$ $=0.2$ exhibits a collapse transition at a packing fraction of $44 \%$, which points to a demixed state. The promotion of a collapse transition for systems with a larger hard-sphere solvent (colloid), corresponding to a decreasing value of $d$, has also been noted. ${ }^{51}$ As we mentioned earlier, the lower AO limit (minimum size of the polymer segment corresponding 


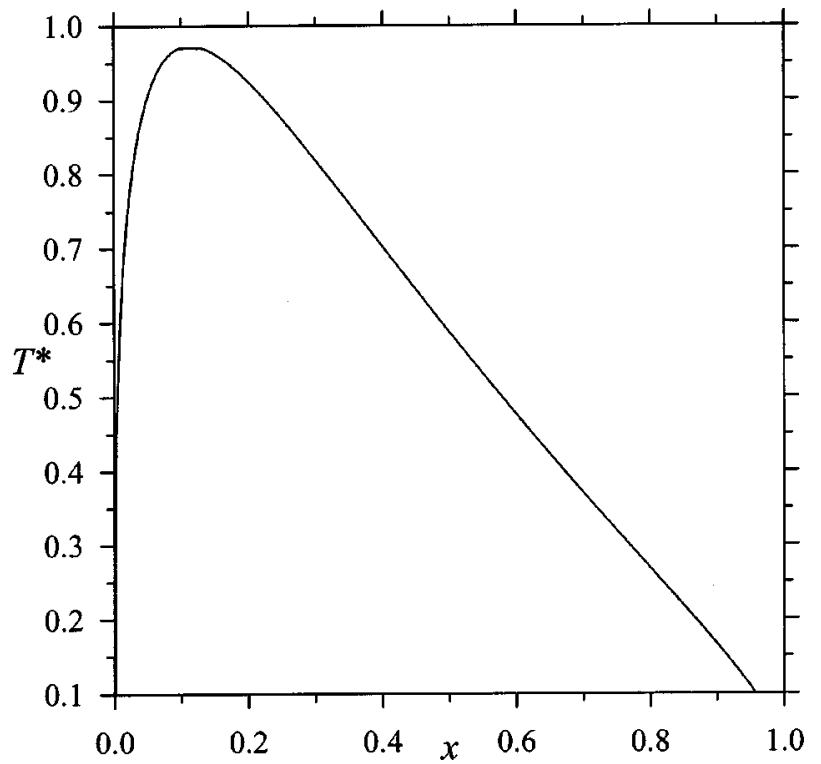

FIG. 5. Temperature-composition phase diagram of the fluid-fluid phase equilibria for an athermal binary mixture of colloids (hard spheres of diameter $\sigma_{C}$ ) and flexible polymers (chains formed from tangent hard spheres of diameter $\sigma_{P}$ ) determined from the Wertheim TPT1 approach. The parameters characterizing the system are a polymer-segment to colloid diameter ratio of $d=\sigma_{P} / \sigma_{C}=0.06$ and a polymer chain length of $m=500$. The reduced temperature is defined as the reciprocal of the reduced pressure $T^{*}$ $=1 / P^{*}=k T /\left(P \sigma_{C}^{3}\right)$, and $x=x_{P}$ represents the mole fraction of the polymers.

to a lower value of $d$ ) is not shown in Fig. 4 because of the lack of a proper stability condition to include the solidification of the polymer-rich phase.

We end our discussion with an examination of the thermodynamics of mixing for the athermal colloid-polymer model. For the purpose of comparison with the fluid-fluid phase equilibria exhibited by simple mixtures, ${ }^{1}$ it is useful to display the phase separation of the colloid-polymer system in the "temperature"-composition representation. A reduced temperature can be defined in terms of the reciprocal of the reduced pressures as $T^{*}=1 / P^{*}=k T /\left(P \sigma_{C}^{3}\right)$, noting that in an athermal system only the temperature-pressure ratio is the important variable. The fluid phase equilibria (binodal curve) for the colloid-polymer mixture with $m=500$ and $d=0.06$, determined by ensuring that the pressure and chemical potentials of each component in each phase are equal, is represented in this fashion in Fig. 5. At temperatures above the upper critical solution temperature (UCST), which correspond to the low-pressure states, the mixed fluid phase of the system is stable. When the temperature is lowered below the UCST (in this case $T_{\mathrm{cr}}^{*}=0.980$, corresponding to increasing the pressure above $P_{\mathrm{cr}}^{*}=1.02$ ), the mixture exhibits fluid-fluid phase separation into colloid-rich and colloid-poor phases. One can now ask the question: What is the main thermodynamic contribution leading to such a fluid phase separation? In order to answer this type of question it is common to determine the thermodynamic functions of mixing. The changes in the Gibbs free energy, entropy, enthalpy, and volume on mixing of the colloid and polymer for a composition $x_{C}$ and $x_{P}$ at a constant pressure $P$ are defined in the usual way as ${ }^{1}$

$$
\begin{aligned}
& \Delta G_{m}(P, T)=G(P, T)-N_{C} G_{C}(P, T)-N_{P} G_{P}(P, T), \\
& \Delta S_{m}(P, T)=S(P, T)-N_{C} S_{C}(P, T)-N_{P} S_{P}(P, T), \\
& \Delta H_{m}(P, T)=H(P, T)-N_{C} H_{C}(P, T)-N_{P} H_{P}(P, T), \\
& \Delta V_{m}(P, T)=V(P, T)-N_{C} V_{C}(P, T)-N_{P} V_{P}(P, T) .
\end{aligned}
$$

The Gibbs free energy of mixing of the colloid-polymer system with $m=500$ and $d=0.06$ is shown in Fig. 6(a) for a temperature $T^{*}=1.25\left(P^{*}=0.8\right)$ above and a temperature $T^{*}=0.833\left(P^{*}=1.2\right)$ below the critical point. In the case of the $P^{*}=0.8$ state (above the UCST) the Gibbs free energy of mixing is always negative and does not exhibit a change in curvature over the entire composition range, indicating a stable miscible mixture. For the state $P^{*}=1.2$ (below the UCST), the Gibbs function remains negative, but a change in curvature can be seen in the colloid-rich region of the diagram; the second derivative of the Gibbs free energy with composition becomes negative in this region, indicating an instability in the fluid mixture which leads to demixing (the spinodal is the point at which the curvature is first seen to become negative). The entropy of mixing of the colloidpolymer system for these two (mixed and demixed) states is represented in Fig. 6(b). The first rather surprising observation is that the entropy of mixing is always positive for the athermal colloid-polymer system; for the mixed state $\left(P^{*}=0.8\right)$, it is virtually indistinguishable from the ideal entropy of mixing, and though the entropy of mixing is seen to drop for the demixed state $\left(P^{*}=1.2\right)$, it is still significantly positive. The consequence of this is that the entropy of mixing in such a system favors the mixed state, and care should be taken when one refers to "entropy-driven" transitions in athermal colloid-polymer systems. So what is responsible for the phase separation? The answer of course lies in the enthalpy of mixing which constitutes the other contribution to the free energy of a system at constant pressure ( $\Delta G_{m}=\Delta H_{m}-T \Delta S_{m}$ ). It is clear from Fig. 6(c) that the enthalpy of mixing is large (in comparative terms) and positive over the entire composition range both for the mixed and demixed states, with a slight asymmetry in the curves towards the colloid-rich part of the diagram. The importance of such an enthalpic contribution to the free energy in these athermal mixtures has already been recognized by Honnell and Hall. ${ }^{63}$ As the temperature is lowered (or in this case the pressure increased), the contribution of the enthalpy to the Gibbs free energy of mixing dominates over the entropic contribution and mixing becomes unstable relative to fluid phase separation. This is the same mechanism that is responsible for liquid-liquid immiscibility in simple mixtures (cf., Fig. 5). The positive contribution of the enthalpy of mixing gives rise to the change in curvature of the Gibbs free energy in the colloid region of the diagram and to the subsequent phase separation (common tangent condition). What is the reason for this large positive enthalpy of mixing in athermal colloid-polymer systems? The change in the internal energy of an athermal system is zero $\left(\Delta U_{m}=0\right)$, so the only contribution to the enthalpy is due to the change in volume of mixing $\left(\Delta H_{m}=\Delta U_{m}+P \Delta V_{m}=P \Delta V_{m}\right)$. This means that the volume of mixing in the system must be positive, as can 

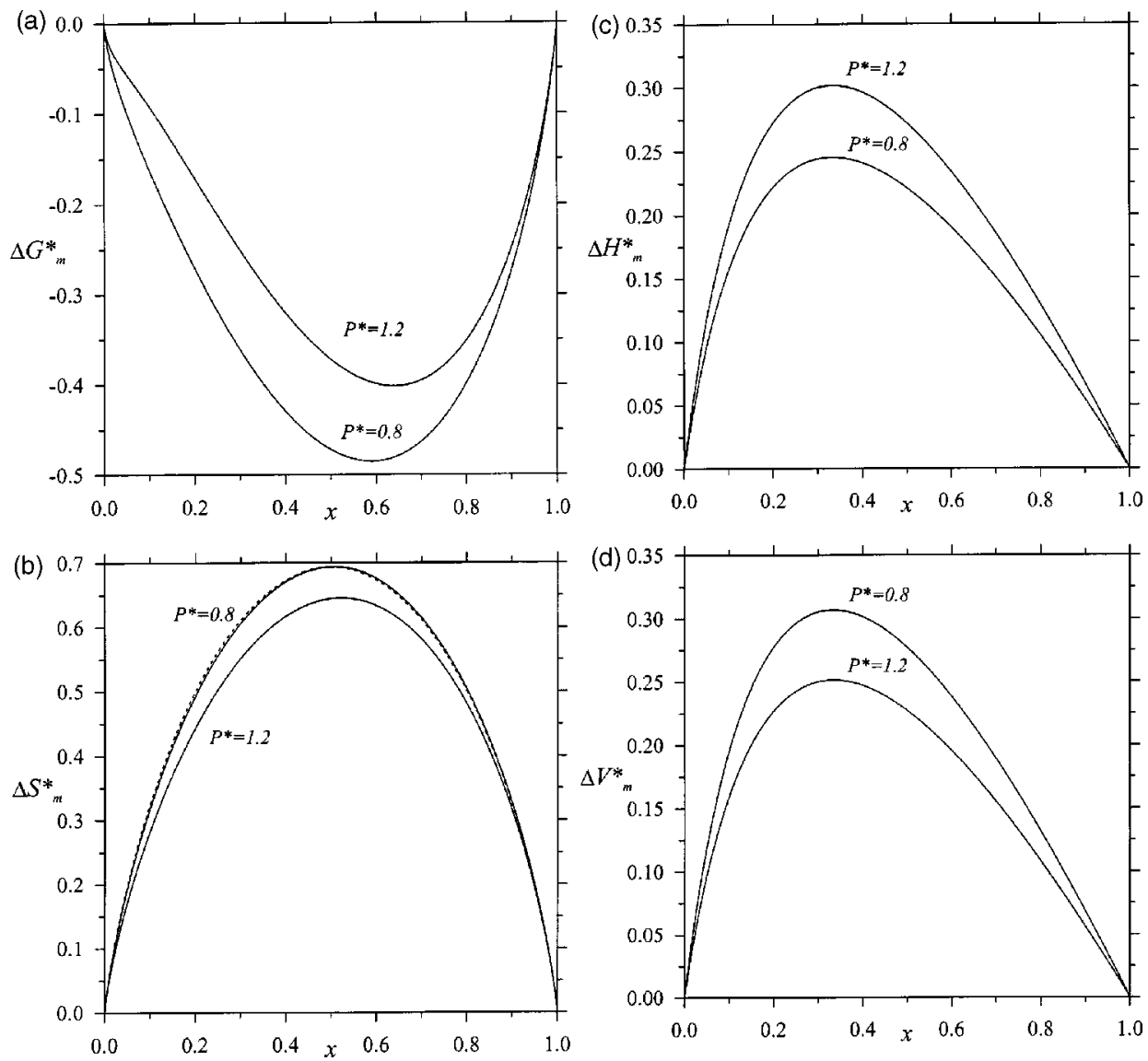

FIG. 6. Thermodynamic properties of mixing for an athermal binary mixture of colloids (hard spheres of diameter $\sigma_{C}$ ) and flexible polymers (chains formed from tangent hard spheres of diameter $\sigma_{P}$ ) determined from the Wertheim TPT1 approach. The parameters characterizing the system are a polymer-segment to colloid diameter ratio of $d=\sigma_{P} / \sigma_{C}=0.06$ and a polymer chain length of $m=500$. The reduced Gibbs free energy $\Delta G_{m}^{*}$ $=\Delta G_{m} /(N k T), \quad(\mathrm{b})$ entropy $\Delta S_{m}^{*}$ $=\Delta S_{m} /(N k), \quad$ (c) enthalpy $\Delta H_{m}^{*}$ $=\Delta H_{m} /(N k T)$, and (d) volume $\Delta V_{m}^{*}$ $=\Delta V_{m} / \sigma_{C}^{3}$ of mixing are plotted for mixed $\left(P^{*}=0.8\right)$ and a demixed $\left(P^{*}\right.$ $=1.2)$ states; the ideal entropy of mixing is also shown as a dashed curve. The reduced pressure is defined as $P^{*}=\beta P \sigma_{C}^{3}$, and $x=x_{P}$ represents the mole fraction of the polymers. indeed be seen from Fig. 6(d). The positive volume of mixing leads to a positive enthalpy of mixing, which in turn makes an unfavorable contribution to the Gibbs free energy of mixing. The large volume of mixing in the system is due to large colloid-polymer excluded volume interactions: when the colloid and polymer are mixed at constant volume the increase in the excluded volume interactions leads to a higher pressure; in order to maintain the pressure constant, the mixture will tend to increase its volume and minimize the unfavorable excluded volume interactions. Of course, the maximization of the free volume (minimization of the excluded volume) is an entropic effect, but as we have shown for the athermal colloid-polymer system this leads to an unfavorable enthalpy of mixing not an unfavorable entropy of mixing. The Gibbs function represents all the entropic contributions of the system and its surroundings, and every spontaneous process is driven by an increase in the total entropy. When the diameter of the polymer segment is increased relative to the diameter of the colloid (corresponding to an increase in the ratio $d$ ), the volume of mixing decreases and eventually becomes negative. For the system with $m=500$ the volume of mixing becomes negative for ratios above $d \approx 0.24$; this confirms that the symmetric system with $d=1$ has a negative volume of mixing as was found by Honnell and Hall. ${ }^{63}$ The explanation for the negative volume of mixing and lack of fluid phase separation for the systems with a larger $d$ is that as the polymer segments are made larger, the magnitude of the polymer-polymer excluded volume increases relative to polymer-colloid excluded volume which drives the demixing.

\section{CONCLUSIONS}

In this work we have presented the results of a study of the molecular conditions which give rise to fluid phase separation in binary mixtures of hard colloids and flexible polymers. By using the Wertheim TPT1 theory, it is possible to treat both components at the "microscopic" level of the segments making up the polymer; i.e., not only the length of the polymer, but the diameter of the segment is a relevant variable. As well as a description of the colloid-colloid and colloid-polymer interactions, this approach allows for the excluded volume effect between polymers to be taken into consideration. One advantage of the TPT1 approach is that one does not explicitly require any information about the dimension of the polymer, which can change abruptly both with density and composition. The incorporation of polymer-polymer interactions leads to significant differences with the theoretical predictions for ideal chains, not only in the protein limit (large polymers and small colloids), but also in the AO limit (polymers which are much smaller than the colloids). In common with other recent studies, ${ }^{42,45}$ we find that the treatment of the polymer-polymer interactions suggest that the critical density for fluid phase separation tends to a finite value as the chain length is increased. In addition, there also appears to be a maximum in the polymer segment-colloid diameter ratio of about $d=\sigma_{P} / \sigma_{C} \approx 0.2$, above which the athermal colloid-polymer system is not expected to exhibit fluid phase separation regardless of the chain length of the polymer. We also show that an unfavorable enthalpy of mixing (due to the large colloid-polymer 
excluded volume interaction) is responsible for the fluid phase separation in our athermal colloid polymer system; the entropy of mixing in this case is always positive and favors a mixed state, and a more careful use of the term "entropydriven" phase transition is recommended for such systems. In the case of polymer solutions the segments making up the polymer are of a similar size to the solvent and attractive interactions play a crucial role. The mechanism for fluid phase separation is completely different in polymer solutions: the volume of mixing can be negative in regions of the phase diagram corresponding to dilute solutions of polymer, which gives rise to a negative (unfavorable) entropic contribution. ${ }^{55}$ We hope that our analysis of the nature of fluid phase separation in colloid-polymer systems has provided a slightly different perspective to this well-studied area. In future work, we plan to incorporate both the effect of the attractive interactions and polydispersity within a Wertheim TPT1 approach to make quantitative comparisons with experimental measurements of phase separation in colloidpolymer mixtures. An attempt will also be made to treat the solid phases in such systems.

\section{ACKNOWLEDGMENTS}

P.P. would like to thank the Modelling Program of BP Chemicals for funding a studentship. S.V. would like to thank the ROPA program of the Engineering and Physical Sciences Research Council (EPSRC) for a research fellowship (Grant No. GR/N03358). We acknowledge further support from the Joint Research Equipment Initiative (JREI) of the EPSRC for computer hardware (Grant No. GR/M94427) and the Royal Society-Wolfson Foundation for the award of a refurbishment grant.

${ }^{1}$ J. S. Rowlinson and F. L. Swinton, Liquids and Liquid Mixtures, 3rd ed. (Butterworth Scientific, London, 1982).

${ }^{2}$ D. Frenkel, Physica A 263, 26 (1999); 313, 1 (2002).

${ }^{3}$ T. Biben and J.-P. Hansen, Phys. Rev. Lett. 66, 2215 (1991).

${ }^{4}$ H. N. W. Lekkerkerker and A. Stroobants, Physica A 195, 387 (1993).

${ }^{5}$ C. Caccamo and G. Pellicane, Physica A 235, 149 (1997).

${ }^{6}$ M. Dijkstra, R. van Roij, and R. Evans, Phys. Rev. Lett. 81, 2268 (1998); Phys. Rev. E 59, 5744 (1999).

${ }^{7}$ J. A. Cuesta, Europhys. Lett. 46, 197 (1999).

${ }^{8}$ P. B. Warren, Europhys. Lett. 46, 295 (1999).

${ }^{9}$ R. P. Sear, Europhys. Lett. 44, 531 (1998).

${ }^{10}$ W. C. K. Poon and P. N. Pusey, in Observation, Prediction and Simulation of Phase Transitions in Complex Fluids, edited by M. Baus, L. F. Rull, and J. Ryckaert (Kluwer, Dordrecht, 1995).

${ }^{11}$ F. K. R. Li-In-On, B. Vincent, and F. A. Waite, ACS Symp. Ser. 9, 165 (1975).

${ }^{12}$ F. L. Calderon, J. Bibette, and J. Bais, Europhys. Lett. 23, 653 (1993).

${ }^{13}$ P. N. Pusey, A. D. Pirie, and W. C. K. Poon, Physica A 201, 322 (1993); P. N. Pusey, W. C. K. Poon, S. M. Ilett, and P. Bartlett, J. Phys.: Condens. Matter 6, A29 (1994); S. M. Ilett, A. Orrock, W. C. K. Poon, and P. N. Pusey, Phys. Rev. E 51, 1344 (1995); W. C. K. Poon, A. D. Pirie, and P. N. Pusey, Faraday Discuss. 101, 65 (1995); A. Moussaid, W. C. K. Poon, P. N. Pusey, and M. F. Soliva, Phys. Rev. Lett. 82, 225 (1999).

${ }^{14}$ F. Renth, W. C. K. Poon, and R. M. L. Evans, Phys. Rev. E 64, 031402 (2001).

${ }^{15}$ N. A. M. Verhaegh, J. S. van Duijneveldt, J. K. G. Dhont, and H. N. W. Lekkerkerker, Physica A 230, 409 (1996).

${ }^{16}$ I. Bodnar and W. D. Oosterbaan, J. Chem. Phys. 106, 7777 (1997).

${ }^{17}$ A. D. Dinsmore, A. G. Yodh, and D. J. Pine, Phys. Rev. E 52, 4045 (1995).

${ }^{18}$ R. Verma, J. C. Crocker, T. C. Lubensky, and A. G. Yodh, Phys. Rev. Lett. 81, 4004 (1998).
${ }^{19}$ S. Ramakrishnan, M. Fuchs, K. S. Schweizer, and C. F. Zukoski, J. Chem. Phys. 116, 2201 (2002).

${ }^{20}$ S. B. Zimmerman and A. P. Minton, Annu. Rev. Biophys. Biomol. Struct. 22, 27 (1993).

${ }^{21}$ S. Asakura and F. Oosawa, J. Chem. Phys. 22, 1255 (1954); J. Polym. Sci. 33, 183 (1958).

${ }^{22}$ A. Vrij, Pure Appl. Chem. 48, 471 (1976).

${ }^{23}$ J. F. Joanny, L. Leibler, and P.-G. de Gennes, J. Polym. Sci., Polym. Phys. Ed. 17, 1073 (1979).

${ }^{24}$ E. J. Meijer and D. Frenkel, Phys. Rev. Lett. 67, 1110 (1991); E. J. Meijer and D. Frenkel, J. Chem. Phys. 100, 6873 (1994).

${ }^{25}$ M. Dijkstra, J. M. Brader, and R. Evans, J. Phys.: Condens. Matter 11, 10079 (1999).

${ }^{26}$ A. Hanke, E. Eisenriegler, and S. Dietrich, Phys. Rev. E 59, 6853 (1999).

${ }^{27}$ A. P. Gast, C. K. Hall, and W. B. Russel, J. Colloid Interface Sci. 96, 251 (1983).

${ }^{28}$ B. Vincent, Colloids Surf. 24, 269 (1987); B. Vincent, J. Edwards, S. Emmett, and R. Croot, ibid. 31, 267 (1988).

${ }^{29}$ P. N. Pusey, Colloidal Suspensions. Les Houches: Liquides, Cristallisation et Transition Vitreuse/Liquids, Freezing and Glass Transition (Elsevier, Amsterdam, 1989).

${ }^{30}$ H. N. W. Lekkerkerker, W. C. K. Poon, P. N. Pusey, A. Stroobants, and P. B. Warren, Europhys. Lett. 20, 559 (1992).

${ }^{31}$ P. Bolhuis and D. Frenkel, J. Chem. Phys. 101, 9869 (1994).

${ }^{32}$ H. N. W. Lekkerkerker and B. Widom, Physica A 285, 483 (2000).

${ }^{33}$ S. M. Oversteegen and H. N. W. Lekkerkerker, Physica A 310, 181 (2002).

${ }^{34}$ J. M. Brader and R. Evans, Physica A 306, 287 (2002).

${ }^{35}$ R. P. Sear, Phys. Rev. Lett. 86, 4696 (2001).

${ }^{36}$ M. S. Wertheim, J. Chem. Phys. 87, 7323 (1987).

${ }^{37}$ W. G. Chapman, G. Jackson, and K. E. Gubbins, Mol. Phys. 65, 1057 (1998).

${ }^{38}$ M. Schmidt and M. Fuchs, J. Chem. Phys. 117, 6308 (2002).

${ }^{39}$ M. Fuchs and K. S. Schweizer, J. Phys.: Condens. Matter 14, R239 (2002).

${ }^{40}$ P. B. Warren, S. M. Ilett, and W. C. K. Poon, Phys. Rev. E 52, 5205 (1995).

${ }^{41}$ M. Fuchs and K. S. Schweizer, Europhys. Lett. 51, 621 (2000); Phys. Rev. E 64, 021514 (2001).

${ }^{42}$ R. P. Sear, Phys. Rev. E 66, 051401 (2002).

${ }^{43}$ P. G. Bolhuis, E. J. Meijer, and A. A. Louis, Phys. Rev. Lett. 90, 068304 (2003).

${ }^{44}$ A. A. Louis, P. G. Bolhuis, J.-P. Hansen, and E. J. Meijer, Phys. Rev. Lett. 85, 2522 (2000); P. G. Bolhuis, A. A. Louis, J.-P. Hansen, and E. J. Meijer, J. Chem. Phys. 114, 4296 (2001); P. G. Bolhuis, A. A. Louis, and J.-P. Hansen, Phys. Rev. E 64, 021801 (2001); P. G. Bolhuis and A. A. Louis, Macromolecules 35, 1860 (2002).

${ }^{45}$ P. G. Bolhuis, A. A. Louis, and J.-P. Hansen, Phys. Rev. Lett. 89, 128302 (2001).

${ }^{46}$ D. G. A. L. Aarts, R. Tuinier, and H. N. W. Lekkerkerker, J. Phys.: Condens. Matter 14, 7551 (2002).

${ }^{47}$ W. H. Stockmayer, Macromol. Chem. Phys. 35, 54 (1960).

${ }^{48}$ I. Nishio, S. T. Sun, G. Swislow, and T. Tanaka, Nature (London) 281, 208 (1979); G. Swislow, S. T. Sun, I. Nishio, and T. Tanaka, Phys. Rev. Lett. 44, 796 (1980).

${ }^{49}$ P.-G. de Gennes, Scaling Concepts in Polymer Physics (Cornell University Press, Ithaca, NY, 1979).

${ }^{50}$ M. Dijkstra and D. Frenkel, Phys. Rev. Lett. 72, 298 (1994); M. Dijkstra, D. Frenkel, and J.-P. Hansen, J. Chem. Phys. 101, 3179 (1994).

${ }^{51}$ G. Luna-Bárcenas, G. E. Bennett, I. C. Sanchez, and K. P. Johnston, J. Chem. Phys. 104, 9971 (1996).

${ }^{52}$ F. A. Escobedo and J. J. de Pablo, Mol. Phys. 89, 1733 (1996).

${ }^{53}$ C. J. Grayce, J. Chem. Phys. 106, 5171 (1997).

${ }^{54}$ M. P. Taylor and J. E. G. Lipson, Fluid Phase Equilib. 150-151, 641 (1998).

${ }^{55}$ P. Paricaud, A. Galindo, and G. Jackson (unpublished).

${ }^{56}$ J. K. Suen, F. A. Escobedo, and J. J. de Pablo, J. Chem. Phys. 106, 1288 (1997).

${ }^{57}$ W. Bruns and R. Bansal, J. Chem. Phys. 74, 2064 (1981); 74, 5149 (1981).

${ }^{58}$ S. Toxvaerd, J. Chem. Phys. 86, 3667 (1987).

${ }^{59}$ B. Smit, A. van der Put, C. J. Peters, J. de Swaan Arons, and J. P. J. Michels, Chem. Phys. Lett. 144, 555 (1988).

${ }^{60}$ G. Luna-Bárcenas, D. G. Gromov, J. C. Meredith, I. C. Sanchez, J. J. de Pablo, and K. P. Johnston, Chem. Phys. Lett. 278, 302 (1997).

${ }^{61}$ J. M. Polson and M. J. Zuckerman, J. Chem. Phys. 116, 7244 (2002).

${ }^{62}$ M. G. Moro, N. Kern, and D. Frenkel, Europhys. Lett. 48, 332 (1999). 
${ }^{63}$ R. Dickman and C. K. Hall, J. Chem. Phys. 89, 3168 (1988); K. G. Honnell and C. K. Hall, ibid. 95, 4481 (1991).

${ }^{64}$ S. Varga, A. Galindo, and G. Jackson, J. Chem. Phys. 117, 7207 (2002).

${ }^{65}$ T. Boublík, J. Chem. Phys. 53, 471 (1970).

${ }^{66}$ G. A. Mansoori, N. F. Carnahan, K. E. Starling, and T. W. Leland, J. Chem. Phys. 54, 1523 (1971).

${ }^{67}$ G. Jackson, J. S. Rowlinson, and F. van Swol, J. Phys. Chem. 91, 4907 (1987).

${ }^{68}$ N. F. Carnahan and K. E. Starling, J. Chem. Phys. 51, 635 (1969).

${ }^{69}$ M. S. Wertheim, J. Stat. Phys. 35, 19 (1984); 35, 35 (1984); 42, 459 (1986); 42, 477 (1986).

${ }^{70}$ A. Ben-Naim, J. Chem. Phys. 54, 1387 (1971).

${ }^{71}$ L. R. Pratt and D. Chandler, J. Chem. Phys. 67, 3683 (1977).
${ }^{72}$ T. Boublík, Mol. Phys. 59, 775 (1986).

${ }^{73}$ P. T. Cummings and G. Stell, Mol. Phys. 60, 1315 (1987); Y. Zhou and G. Stell, J. Chem. Phys. 96, 1507 (1992).

${ }^{74}$ E. Kierlik and M. L. Rosinberg, J. Chem. Phys. 97, 9222 (1992).

${ }^{75}$ D. Ghonasgi, M. Llano-Restrepo, and W. G. Chapman, J. Chem. Phys. 98, 5662 (1993).

${ }^{76}$ T. Boublík, Mol. Phys. 68, 191 (1989); T. Boublík, C. Vega, and M. Diaz Peña, J. Chem. Phys. 93, 730 (1990); C. Vega, J. M. Labaig, L. G. MacDowell, and E. Sanz, ibid. 113, 10398 (2000).

${ }^{77}$ D. C. Williamson and G. Jackson, Mol. Phys. 86, 819 (1995).

${ }^{78}$ C. Vega and L. G. MacDowell, J. Chem. Phys. 114, 10411 (2001).

${ }^{79}$ C. Vega, J. Chem. Phys. 108, 3074 (1998). 